\title{
Qualitative Scientific Modeling and Loop Analysis ${ }^{1}$ \\ James Justus \\ University of Texas at Austin \\ justus.phil@mail.utexas.edu
}

$$
\text { 9/13/04 }
$$

\begin{abstract}
Loop analysis is a method of qualitative modeling anticipated by Sewall Wright and systematically developed by Richard Levins. In Levins' (1966) distinctions between modeling strategies, loop analysis sacrifices precision for generality and realism. Besides criticizing the clarity of these distinctions, Orzack and Sober (1993) argued qualitative modeling is conceptually and methodologically problematic. Loop analysis of the stability of ecological communities shows this criticism is unjustified. It presupposes an overly narrow view of qualitative modeling and underestimates the broad role models play in scientific research, especially in helping scientists represent and understand complex systems.
\end{abstract}

1. Introduction. Levins (1966) claimed scientific modeling can maximize at most two of three virtues: generality, realism, and precision. Models that sacrifice generality (SG) make precise quantitative predictions about specific systems and maximize realism by capturing as many system details as possible. Models that sacrifices realism (SR) make unrealistic assumptions so scientists can describe systems with general, mathematicallytractable, equations that produce precise quantitative predictions.

Qualitative modeling sacrifices precision for realism and generality by abandoning quantitative accuracy and focusing on qualitative relations between model variables. Loop analysis, one method of qualitative modeling systematically developed by Levins (1974, 1975, 1998; Puccia and Levins 1985), consists of the analysis of signed digraphs (directed graphs) representing whether increases in one variable induce qualitative increases or decreases in other variables, or leave them unchanged.

\footnotetext{
${ }^{1}$ Thanks to Carl Salk and Sahotra Sarkar for comments.
} 
Levins (1966) did not explicitly define 'generality', 'realism', and 'precision', which prompted Orzack and Sober (1993) to criticize the clarity of his distinctions between modeling strategies. They also criticized that:

(i) the grounds for accepting qualitative predictions are often unstated; and,

(ii) unlike quantitative testing, qualitative testing cannot determine how well models account for data.

This paper responds to (i)-(ii) by analyzing the loop analysis of the stability of ecological communities. Section 2 briefly considers some weaknesses of SG and SR modeling of complex systems that qualitative modeling avoids. After defining Lyapunov stability, Section 3 analyzes how loop analysis can be used to evaluate the stability of ecological communities. Based on this analysis, Section 4 argues Orzack and Sober's (1993) claim that qualitative modeling is methodologically and conceptually problematic is unwarranted. Specifically, the basis of (i) as a criticism of qualitative modeling is dubious, and (ii) presupposes an overly narrow conception of qualitative modeling and unjustifiably limited view of the function of models within science.

2. Weaknesses of Quantitative Modeling of Complex Systems. For Levins, successful scientific research requires several different modeling strategies, each exhibiting strengths and weaknesses relative to different purposes and contexts. In the context of modeling complex systems to understand their dynamics, Levins (1966) thought SG and SR modeling, unlike qualitative modeling, faces significant difficulties.

Levins $(1966,421)$ considered three disadvantages: 
(i) constructing SG models requires measurement of an intractable number of parameters, many requiring several years to measure precisely;

(ii) even if these parameters could be measured, the resulting differential (difference) equations would be analytically insoluble and exhaust the numerical solution capabilities of computers; and,

(iii) even if soluble, the solutions, "would have no meaning for us."

Reason (i) refers to the pragmatic problem of measuring the numerous parameters required to represent as many features of complex systems as possible and thereby maximize model realism. The vast amount of data this requires is usually unavailable, especially within the monetary and temporal constraints of ecological research. Reason (ii) refers to the unmanageability of such complicated models. The sheer size and intractability of models maximizing realism and precision made Levins (1966) skeptical SG models would provide scientifically useful representations of complex systems. ${ }^{2}$ Reason (iii) refers to the limited explanatory power of SG models. Complicated models that are difficult to manage are also difficult to comprehend. Without a clear comprehension of their structure and dynamics, SG models often frustrate rather than enhance understanding of the phenomena they represent. ${ }^{3}$ The ecologist Daniel Botkin emphasized this problem in his criticism of the results of the "systems approach" to ecosystem modeling which, historically, was the target of Levins' (1966) criticisms of SG modeling: "Models are useful when they demonstrate clearly the implications of

\footnotetext{
${ }^{2}$ Advances in computation and simulation techniques have mitigated this difficulty and Levins' (1998) recent work on loop analysis does not make this claim.

${ }^{3}$ As stated, (iii) is probably too strong. It entails solutions found by numerical simulation for complex physical systems are completely incomprehensible, which is implausible given their importance in physics (Winsberg 2001).
} 
assumptions, but the implications of manipulating a great many vaguely based parameters cannot be clear" $(1977,385)$.

SR models of complex systems face different disadvantages. Unlike SG models, SR models focus on small sets of salient system components and avoid (i)-(iii) above. This restricted focus is achieved through idealization, ignoring some system components and interactions, treating interactions as instantaneous, representing discrete components with continuous variables, and so on. Scientists can then approximate real-world systems with thoroughly studied, well-understood mathematical models that produce quantitative predictions. The underlying assumption, Levins (1966) suggested, is that differences between predictions of idealized models and observations will help identify what idealizations preserve accurate description. ${ }^{4}$

The cost of unrealistic idealizations, however, is uncertainty about whether modeling results demonstrate properties of the represented system or are byproducts of unrealistic idealizations. Since it is often unclear what components are primarily responsible for a system's dynamics, unrealistic idealizations may significantly mischaracterize its most important properties. Consequently, an enhanced sense of understanding conveyed by a SR model may fail to be about the system it is intended to represent. This especially troubled Levins since he believed biologists often uncritically emulated mathematically sophisticated models of physics to ensure their modeling is mathematically rigorous, with the consequence that, "theoretical work often diverged too far from life and became exercises in mathematics inspired by biology rather than an

\footnotetext{
${ }^{4}$ Wimsatt's (1987) analysis of the development of "chromosomal mechanics" within the Morgan school provides an excellent account of how this motivation can translate into scientific methodology.
} 
analysis of living systems" (1968, 4). Loop analysis, Section 3 shows, achieves mathematical rigor without sacrificing realism.

3. Qualitative Stability Criteria and Loop Analysis. Consider a system represented by $n$ differential equations:

$$
\frac{d x_{i}(t)}{d t}=F_{i}\left(x_{1}, \ldots, x_{k}, \ldots, x_{n} ; c_{1}, \ldots, c_{m}\right)
$$

where $c_{j}$ are parameters, $x_{k}$ are variables, $1 \leq j \leq m$, and $1 \leq i, k \leq n$. At an equilibrium $\mathbf{x}^{*}$, $(\forall i) \frac{d x_{i}(t)}{d t}=0$.

In general, a system at equilibrium can be globally or locally stable. Informally, a system at a globally stable equilibrium returns to equilibrium following any disturbance. It is extremely improbable any ecological systems are globally stable, but probable some are locally stable. ${ }^{5}$ Local stability depends upon how systems behave in the neighborhood of an equilibrium. To analyze this behavior, the equations of (1) can be linearized:

$$
\frac{d \mathbf{x}(t)}{d t}=\mathbf{A} \mathbf{x}(t)
$$

where $\mathbf{x}(t)$ is the vector of $n$ variables $x_{1}, \ldots, x_{n}$ and $\mathbf{A}$ is the $n \times n$ Jacobian matrix of constant real coefficients $a_{i j}$ derived from the non-linearized interaction terms $c_{i j}=\frac{\partial F_{i}}{\partial x_{j}}$.

By definition, an equilibrium $\mathbf{x}^{*}$ is Lyapunov stable iff:

$$
(\forall \varepsilon>0)(\exists \delta>0)\left(\left|\mathbf{x}\left(t_{0}\right)-\mathbf{x}^{*}\right|<\delta \Rightarrow\left(\forall t \geq t_{0}\right)\left(\left|\mathbf{x}(t)-\mathbf{x}^{*}\right|<\varepsilon\right)\right) ;
$$

where $\mathbf{x}(t)$ is a solution of (2) with initial conditions $\mathbf{x}\left(t_{0}\right)$ and brackets designate a distance metric. Informally, (3) says $\mathbf{x}^{*}$ is Lyapunov stable if a system beginning in a

\footnotetext{
${ }^{5}$ Lewontin (1969) analyzed the distinction between local and global stability in detail.
} 
neighborhood of $\mathbf{x}^{*}$ remains in it after small perturbations. By definition, $\mathbf{x}^{*}$ is asymptotically Lyapunov stable iff (3) and $\mathbf{x}(t) \rightarrow \mathbf{x}^{*}$ as $t \rightarrow \infty$.

Whether $\mathbf{x}^{*}$ is asymptotically Lyapunov stable (hereafter 'stable') depends upon the eigenvalues of $\mathbf{A}$. These are scalar values such that $\operatorname{det}(\mathbf{A}-\lambda \mathbf{I})=0$, the roots of the characteristic polynomial of A. Lyapunov ([1892] 1992) proved $\mathbf{x}^{*}$ is stable iff:

$$
\operatorname{Re} \lambda_{i}(\mathbf{A})<0 \text { for } i=1, \ldots, n \text {; }
$$

where $\operatorname{Re} \lambda_{i}(\mathbf{A})$ designates the real part of $\lambda_{i}$, the ith eigenvalue of $\mathbf{A}$.

The Routh-Hurwitz stability criterion (Gantmacher 1960) extends Lyapunov's theorem and provides the basis for Levins' account of stability in loop-theoretic terms. It states (4) holds iff every Hurwitz determinant $\mathbf{H}_{i}(i=1, \ldots, n)$ is positive, where $\mathbf{H}_{i}=\left|\begin{array}{ccccc}b_{1} & b_{3} & b_{5} & \cdots & b_{2 i-1} \\ b_{0} & b_{2} & b_{4} & \cdots & b_{2 i-2} \\ 0 & b_{1} & b_{3} & \cdots & b_{2 i-3} \\ \vdots & \vdots & \vdots & \ddots & \vdots \\ 0 & 0 & 0 & & b_{i}\end{array}\right|, \quad b_{i}$ are coefficients of the characteristic polynomial $\operatorname{det}(\mathbf{A}-\lambda \mathbf{I})$, and $b_{i}=0$ for $i>n$.

In models of ecological communities, $a_{i j}$ from (2) represents the effect of species $j$ on species $i$. Its quantitative value represents the effects' magnitude and its sign represents whether the effect is positive or negative. Unfortunately, Lyapunov's theorem and the Routh-Hurwitz criterion provide little help in assessing whether ecological communities are stable. The problem is that determining that their conditions are satisfied usually requires information about the quantitative values of the $a_{i j}$, which is typically unavailable and cannot be feasibly obtained. Measuring all these coefficients, for instance, requires measurement of the quantitative effect of $n^{2}$ species interactions each 
of which would involve numerous manipulative experiments. ${ }^{6}$ Coefficient signs are more easily determined. For instance, for competitors, $a_{i j}, a_{j i}<0$ and for predator and prey, $a_{i j}>0$ and $a_{j i}<0$.

Since coefficient signs often can be determined, hypotheses about ecological communities often can be assessed without quantitative data. One such hypothesis is whether the stability of ecological communities depends on the quantitative strengths of species interactions or stems from their qualitative structure in the community. Whereas SG and SR modeling utilizes quantitative data which is unavailable in this context, the sacrifice of quantitative precision makes qualitative modeling well-suited to analyze this hypothesis.

Loop analysis is based on an equivalence between matrices of constant coefficients and signed digraphs, first anticipated by Wright (1921). For systems represented by (2), $x_{1}, \ldots, x_{n}$ correspond to digraph vertices. If $\mathbf{A}$ represents qualitative information about species interactions, $a_{i j}$ take values $+1,-1$, or 0 to represent positive, negative, or null effects of species $j$ on species $i$. These values determine what vertices are connected by edges and the edges' direction and effect. If $a_{i j}=1, x_{j} \rightarrow x_{i}$ designates a positive effect; if $a_{i j}=-1, x_{j} \longrightarrow x_{i}$ designates a negative effect; if $a_{i j}=0$, no edge exists. To illustrate, the matrix for species N, P, and $\mathrm{H},\left[\begin{array}{lll}a_{N N} & a_{N H} & a_{N P} \\ a_{H N} & a_{H H} & a_{H P} \\ a_{P N} & a_{P H} & a_{P P}\end{array}\right]=\left[\begin{array}{ccc}-1 & -1 & +1 \\ +1 & -1 & -1 \\ 0 & +1 & 0\end{array}\right]$ corresponds to the digraph:

\section{[Figure 1]}

\footnotetext{
${ }^{6}$ The extensive exclusion experiments utilized in one of the first such quantitative measurements, in this case between a small set of competing desert rodent and ant species (Brown and Davidson 1977), helps indicate the magnitude of the difficulty.
} 
A loop is series of directed edges from one vertex to itself that crosses no any intermediate variables more than once. The number of edges in a loop is its length and disjunct loops share no vertices. $a_{\mathrm{HP}} a_{\mathrm{PH}}$ and $a_{\mathrm{NN}}$, for instance, are disjunct loops of length 2 and 1.

The equivalence between matrices and digraphs entails a correspondence between product terms of matrix determinants and products of loops. For example, if $\mathbf{A}=\left[\begin{array}{ll}a_{11} & a_{12} \\ a_{21} & a_{22}\end{array}\right], \operatorname{det}(\mathbf{A})=a_{11} a_{22}-a_{12} a_{21}$, which is the difference between the product of length 1 loops and the product of length 2 loops in the digraph of A. Levins $(1975,20)$ generalized this result to $n$-order matrices:

$$
\operatorname{det}_{n}(\mathbf{A})=\sum_{m=1}^{n}(-1)^{n-m} \sum_{L(m, n) \in \mathbf{L}_{m, n}} L(m, n)
$$

where $L(m, n)$ is the product of $n$ coefficients forming $m$ disjunct loops and $\mathbf{L}_{m, n}$ is the set of all such products in the digraph under consideration. $L(2,4)$, for instance, is the product of the four coefficients of two disjunct loops. With this generalization, Levins $(1975,21)$ defined "feedback at level $k$ " in $n$-variable systems:

$$
\mathrm{F}_{k}(\mathbf{A})=\sum_{m=1}^{k}(-1)^{m+1} \sum_{L(m, k) \in \mathbf{L}_{m, k}} L(m, k)
$$

where $1 \leq k \leq n$. Notice $\mathrm{F}_{1}(\mathbf{A})=\sum_{i=1}^{n} a_{i i}$, the sum of the diagonal elements of $\mathbf{A}$, the length 1 loops.

According to Puccia and Levins (1985), feedback is a process by which changes in variables induce changes in other variables that then affect the variables originally changed. Positive feedback enhances change: increase in variables induces further 
increase, and decrease induces further decrease. Negative feedback counteracts change: increase induces decrease, and decrease induces increase. ${ }^{7}$

With this definition of feedback, Levins formulated the Routh-Hurwitz criterion in loop-theoretic terms. ${ }^{8}$ An $n$-variable system is stable iff $(\forall k<n)\left(\mathrm{F}_{k}<0\right)$, and $\left|\begin{array}{ccccc}-F_{1} & -F_{3} & -F_{5} & \cdots & -F_{2 n-1} \\ -F_{0} & -F_{2} & -F_{4} & \cdots & -F_{2 n-2} \\ 0 & -F_{1} & -F_{3} & \cdots & -F_{2 n-3} \\ \vdots & \vdots & \vdots & \ddots & \vdots \\ 0 & 0 & 0 & & -F_{n}\end{array}\right|>0$. The first condition requires negative feedback at every level and the second requires feedback at lower levels to be stronger than feedback at higher levels. ${ }^{9}$

A drawback of this loop-theoretic criterion is that quantitative data is often required to determine whether it is satisfied. The system represented in Figure 1, for example, is stable iff $a_{\mathrm{NN}} a_{\mathrm{HP}}>a_{\mathrm{NP}} a_{\mathrm{HN}}$, which depends on their quantitative values. Furthermore, the probability quantitative data will be required, and the amount of it required, increases with the number of model variables (Dambacher et al. 2003).

This limitation of, ultimately, the Routh-Hurwitz criterion prompted searchs for completely qualitative stability criteria, which economists Quirk and Ruppert (1965) found first. They defined the sign-pattern of a matrix $\mathbf{A}$ as its pattern of coefficients $(+1$, $-1,0)$. $\mathbf{B}$ is sign-similar to $\mathbf{A}$ iff their sign-patterns are identical. $\mathbf{A}$ is sign-stable iff the eigenvalues of every matrix sign-similar to it satisfy (4). That is, sign-stable matrices

\footnotetext{
${ }^{7}$ Levins' understanding of the concept of feedback reveals a peculiar relationship between his scientific commitment to realistic modeling and philosophical view of the status of scientific concepts. Levins intended loop analysis to preserve realism about the system being modeled. He did not believe, however, the feedback utilized in loop analysis designated an objective property of systems (reported in Wimsatt 1970, 252). Rather, Levins thought feedback, and scientific concepts in general, were purely heuristic features of how systems are represented (Cf. Wimsatt 2001).

${ }^{8}$ Details in Puccia and Levins (1985, Ch. 6)

${ }^{9}$ For instance, for 3 variable systems the second condition requires $F_{1} F_{2}+F_{3}>0$.
} 
remain stable with any specification of the quantitative values of their entries that preserves their sign-pattern. They proved a real $n \times n$ matrix $\mathbf{A}=\left[a_{i j}\right]$ is sign-stable iff:

(7) $\quad(\forall i)\left(a_{i i}<0\right)$;

(8) $\quad(\forall i, j)\left[(i \neq j) \Rightarrow\left(a_{i j} a_{j i} \leq 0\right)\right]$;

(9) there are no loops of length $\geq 3$.

Other more complicated necessary and sufficient conditions were found, ${ }^{10}$ but like (7)-(9) these conditions were biologically unrealistic (Jefferies 1974). (7) requires every species be self-damping, which denies the existence of Allee effects, and (8) requires communities not contain competitors or symbionts; both are extraordinarily implausible. It is probable, therefore, that community stability depends on quantitative strengths of species interactions, not only on their qualitative structure in the community.

As this limited exposition indicates, loop analysis of ecological communities is a formally rigorous method of scientific analysis. Establishing (7)-(9) and other conditions entail and are entailed by sign-stability, for instance, requires sophisticated mathematical proof. Before their critique of qualitative modeling, however, Orzack and Sober (1993, 538), characterized a qualitative model as one that, "makes only a qualitative prediction," and, "In this sense, these models are not mathematical." Senses differ about the exact meaning of the term, ${ }^{11}$ but their claim is indefensible if intended as support for the criticism that qualitative modeling, unlike quantitative modeling, is not rigorous because it is not 'mathematical.' Quantification is not necessary for formal rigor in science any more than it is in mathematics. ${ }^{12}$ This point was not lost on Levins (1998), who

\footnotetext{
${ }^{10}$ See Jeffries (1974) and Logofet (1993).

${ }^{11}$ Orzack and Sober (1993) did not provide an account of what makes a model mathematical. Given the previous statement, therefore, it is especially difficult to make sense of their apparently inconsistent claim there are "qualitative mathematical models" (542).

${ }^{12}$ This is especially apparent in the technically sophisticated parts of the social sciences that model the behaviors of rational agents, such as game theory.
} 
considered loop analysis as a type of qualitative mathematics that studies functions specified as only increasing/decreasing, or convex/concave, without quantitative precisification. ${ }^{13}$ Although not explicitly stated, the supposition that rigor requires quantification seems to underlie Orzack and Sober's (1993) criticisms of qualitative modeling addressed in Section $4 .^{14}$

\section{Orzack and Sober's Criticisms of Qualitative Modeling. After criticizing Levins'}

(1966) distinctions between modeling strategies ${ }^{15}$ and concept of robustness, Orzack and Sober (1993) argued qualitative modeling is conceptually and methodologically problematic. Specifically, they criticized that, although qualitative testing can be useful, $(1993,542)$ :

(i) "Grounds for accepting qualitative predictions are often left unstated. One consequence is that investigators sometimes use contradictory criteria to judge the same model...Although this is also a potential problem in quantitative testing, the latter approach usually leads biologists to state test criteria explicitly," and,

\footnotetext{
${ }^{13}$ For Herbert Simon (1991, vi), qualitative analysis is, "the mathematics of monotonic transformations, or, what is nearly the same thing, the mathematics of ordinally measured quantities."

${ }^{14}$ Qualitative modeling can be distinguished from qualitative analysis. The former analyzes models consisting of strictly qualitative relations and assumptions, whereas the latter analyzes the qualitative structure of models consisting of some quantitative features. Since Lyapunov's proof refers to variables ranging over $\mathbf{R}$ and in this sense -even if unstantiated-they are quantitative, one could object that loop analysis of community stability only vindicates qualitative analysis, not qualitative modeling. (I owe this potential objection to Sahotra Sarkar.) This objection, however, does not support Orzack and Sober's criticism for two reasons. First, contrary to their interpretation, Levins (1966) did not believe precision was a dichotomous model attribute (Levins 1993) and, therefore, would think the distinction between quantitative and qualitative and, derivatively, qualitative modeling and analysis was a matter of degree. Second, and most importantly, many other examples of analogously rigorous qualitative modeling do not appeal to real variables or similar quantitative assumptions. Such modeling appeals only, for instance, to ordinal relationships or other qualitative assumptions. In conservation biology see Sarkar and Garson (2004); in the social sciences see Arrow (1984, essays 3 and 6) and Arrow and Raynaud (1986).

${ }^{15}$ For a response see Levins (1993) and Odenbaugh (2003).
} 
(ii) "The most important defect in qualitative testing, however, is that it fails to allow one to answer the most important question about a particular model: How well does that model explain the data?" Qualitative testing may show some models are incompatible with data, but only quantitative testing of quantitative models can determine what one if any sufficiently explains it.

In the context of the adaptationism debate, Orzack and Sober $(1993,543)$ explained that quantitative models providing "sufficient explanations" of traits can be tested with data to determine whether, "natural selection has been so important in a trait's evolution that nonselective forces may be safely ignored," or "natural selection has been an important force in a trait's evolution." Qualitative testing, however, is incapable of such determinations since it, "fails to allow one to discriminate between the claim that natural selection is an important cause of what we observe and the claim that natural selection suffices as an explanation for the trait." Orzack and Sober $(1993,543)$ concluded their criticisms of qualitative optimality models of adaptation with the suggestion, "there is no more compelling reason to reassess the view of models endorsed in Levins' 1966 paper than the fact that the idea of qualitative modeling has hindered the development of an unbiased assessment of the truth [of adaptationism]."

The first problem with these criticisms is that the data required for quantitative testing often do not exist and cannot be collected feasibly. This predicament is not unique to ecology but often true when the dynamics of the system modeled are exceptionally complex. For this reason, Levins stressed the utility of loop analysis in modeling other complex systems, such as social systems (Puccia and Levins 1985). In general, since methods of model construction and testing, for instance model simplification, should 
reflect the, "reality to be described [and] the state of the science" (Levins 1966, 422), comparative criticism of modeling strategies is only justified if both strategies are appropriate for the context. Criticism (ii), therefore, fails to appreciate that qualitative modeling is well-suited to scientific fields in which estimating system parameters and testing quantitative predictions are infeasible.

The second problem is that (i) is dubious as a criticism of qualitative modeling. Although the precise nature of the difficulty is somewhat unclear from their brief discussion, Orzack and Sober (1993) cited (Orzack [1990]) which helps clarify the issue. Orzack (1990) analyzed several studies of an extension of Hamilton's model of mating competition due to Werren (1980). ${ }^{16}$ Werren (1980) tested his model (with subsequent studies following suit) by plotting observations of the second sex ratio of Nasonia vitripennis against predicted values and visually assessing their "fit." Based on this assessment, Werren $(1980,1158)$ judged, "The sex-ratio data show the trend predicted," without presenting any statistical tests in support; subsequent studies with similar results and no statistical tests, however, came to different conclusions. Orzack (1990) correctly identified two problems with this method of model evaluation: visual appearance of fit depends significantly on presentation scale and judgments about what can be concluded from such fit vary dramatically.

As criticisms of qualitative modeling, however, these problems are misplaced. Werren (1980) specified his model's mathematical form, estimated the value of the

\footnotetext{
${ }^{16}$ Werren modified Hamilton's model to allow for variable brood sizes, which was more realistic and made it possible to model the mating strategies of the parasitic wasp Nasonia vitripennis. Werren estimated one of the central model variables, ratio of male to female eggs laid in previously unparasitized hosts, and predicted the ratio for eggs laid in previously parasitized hosts (the "second sex ratio" following Orzack [1990]). Previous studies showed Nasonia vitripennis could detect hosts had been previously parasitized and subsequently laid greater proportions of male eggs than in previously unparasitized hosts (Werren 1980).
} 
variable required to make quantitative predictions, and tested those predictions against quantitative data. In Orzack and Sober's (1993, 535-536) classification of model types, Werren's (1980) model is instantiated and generates quantitative point predictions; it is not qualitative. Orzack's (1990) criticisms, therefore, target only one, obviously flawed, type of qualitative test, visual inspection of fit, applied to a quantitative model.

In fact, Orzack and Sober's (1993) view of qualitative testing seems limited to this kind of test. After (i), which highlights what they believed was a general deficiency of qualitative testing, Orzack and Sober $(1993,542)$ continued, "Qualitative assessment of fit can also be highly dependent upon the manner of graphical presentation." Qualitative testing should not, however, be narrowly identified with problematic visual assessments of fit. As Section 3 and examples in the social sciences make clear, qualitative testing can be a rigorous method of scientific analysis. What Orzack (1990) revealed was bad scientific methodology, not methodological problems with qualitative modeling. Regression analysis is the appropriate test of a significant fit between observations and quantitative predictions, for Werren (1980) nonlinear regression. That regressions were not performed justifies suspicion that the results were not significant and this was the reason Werren (1980) and subsequent studies appealed to visual assessments. Application of poor qualitative tests when quantitative data are available and quantitative statistical tests should be performed does not, however, support a general indictment of qualitative modeling.

The final problem is that (ii) presupposes an unjustifiably narrow view of the role of models within scientific practice. For Orzack and Sober (1993), the principal objective of models is to account for data. They (543) argued, for instance, that the fundamental 
flaw of qualitative optimality models of adaptation is their inability to provide sufficient explanations of traits. ${ }^{17}$ Testing quantitative predictions against observations is a critical part of scientific modeling, but models also function to enhance scientists' understanding of phenomena, which is especially crucial in developing scientific explanations. Qualitative models are particularly useful in this context because, unlike SG models, the understanding they provide is generalizable and, unlike SR models, it reflects realistic assumptions about the system represented. ${ }^{18}$

Most accounts of scientific explanation agree that enhancing understanding is essential to explanation (Cf. Trout 2002) but differ about the kind of understanding required. Without broaching the amply discussed issue of what kind is defensible, it should be noted that Sober takes a broad view of the kinds of understanding involved in scientific explanation, unlike causal accounts that narrowly require understanding of the causal processes underlying phenomena. Sober (1983) cogently argued equilibrium explanations are counterexamples to such causal conceptions. Considering differences between causal and equilibrium explanations of 1:1 sex ratios, Sober $(1983,207)$ argued:

The causal explanation focuses exclusively on the actual trajectory of the population; the equilibrium explanation situates that actual trajectory (whatever it may have been) in a more encompassing structure. It is in this way that equilibrium explanations can be more explanatory than causal explanations even though they provide less information about what the actual cause was. This

\footnotetext{
${ }^{17}$ As they use the term (543), 'sufficient explanation' focuses exclusively on model predictions according with data. That qualitative optimality models assist in understanding adaptation (542-543), was clearly considered independent of whether they sufficiently explain the data.

${ }^{18}$ This is not to discount the role SR models may play in developing realistic models. Wimsatt (1987) convincingly showed models that are, "oversimplified, approximate, incomplete, and in other ways false" (28), are important and perhaps necessary tools in the construction of realistic models. Each function of "false models" he listed, however, involves a clear recognition of their lack of realism. Consequently, the understanding these models provide must be carefully qualified to account for this.
} 
difference arises from the fact that explanations provide understanding, and understanding can be enhanced without providing more details about what the cause was. [original italics] ${ }^{19}$

Sober's argument is supportive rather than contrary to the view that (qualitative) looptheoretic explanations of community stability are scientifically sound. Satisfaction of (7)(9), for example, entails a system is sign-stable, but this is consistent with an infinite number of quantitative specifications of variable values. Loop analysis does not pinpoint a particular "causal scenario" of stable system behavior as actual but does "situate" it within certain qualitative constraints on how system components interact. Why then is Sober critical of qualitative modeling a decade later if, as his argument seems to affirm, loop analysis and other qualitative modeling methods enhance scientists' understanding of phenomena and help them develop scientific explanations? ${ }^{20}$

One answer may be the narrow conception of qualitative modeling and implicit assumption it lacks rigor already discussed. A second may lie in a more general tension between Levins' conception of the principal goal of qualitative modeling and the narrow view of scientific modeling Orzack and Sober's (1993) analysis seems to presuppose. According to Puccia and Levins $(1985,4)$, qualitative modeling, "stresses qualitative understanding as the primary goal, rather than numerical prediction.” This prioritization of understanding over quantitative testing conflicts with the emphasis on the latter in Orzack and Sober's (1993) criticisms of qualitative modeling. They seem to assume enhancing understanding is an important, but secondary virtue of modeling. That

\footnotetext{
${ }^{19}$ For Sober (1983), "encompassing structures" are sets of disjunctions of possible "causal scenarios." Causal explanations specify what scenario is the actual cause, equilibrium explanations do not.

${ }^{20}$ Qualitative modeling encompasses a diverse array of methodologies and objectives. See Fishwick and Luker (1991).
} 
qualitative optimality models, for instance, "play an important role in modern efforts to understand adaptation" (Orzack and Sober 1993, 543), which was mentioned but not elucidated, did not mitigate their general criticism that qualitative modeling has, "hindered the unbiased assessment of the truth" about adaptationism.

The functions of scientific modeling, however, extend beyond quantitative testing. ${ }^{21}$ Loop analysis, for instance, helps pinpoint what model variables should be measured to answer specific questions, identifies plausible hypotheses about system behavior and provides reasons to reject others, and enhances ecologists' understanding of ecological systems with complex dynamics. Consider a few representative examples:

1. If a digraph is not sign-stable (e.g. Figure 1), the feedback equations (6) specify what interaction coefficients stability depends upon and identify qualitative restrictions on their quantitative values. Since most quantitative values cannot be measured feasibly in complex systems, loop analysis helps focus limited resources on measuring those needed to evaluate system stability.

2. Loop analysis provides a method for determining how equilibrium values of model variables will respond to changed conditions (Puccia and Levins 1985, Ch. 3). For example, if climate change reduces the level of $\mathrm{N}$ in Figure 1, the conditions under which equilibrium values of $\mathrm{P}$ or $\mathrm{H}$ will increase or decrease can be determined by loop analysis. This can then be used to identify correlations between variables (Puccia and Levins 1985, Ch. 4). If known correlations differ from predicted ones, model structure can be modified to represent the system more realistically.

\footnotetext{
${ }^{21}$ The essays in Morgan and Morrison (1999) present detailed analyses of some of the numerous functions of models within physics, chemistry, and economics.
} 
3. As Section 3 discussed, the biological implausibility of (7)-(9), especially for complex communities, indicates the quantitative strengths of species interactions, rather than their qualitative structure alone, are crucial components of community stability.

Examples of these kinds show how qualitative modeling assists in scientific research by broadly contributing to better representation and understanding of modeled systems. Quantitative testing is a prime, but not necessary, objective of scientific modeling. That qualitative modeling focuses on different but critically important objectives does not indicate a conceptually or methodologically problematic status. 


\section{References}

Arrow, Kenneth J. (1984), Collected Papers. Vol. 3. Cambridge: Harvard University Press.

---------- and Hervé Raynaud (1986), Social Choice and Multicriterion Decision-Making. Cambridge: MIT Press.

Botkin, Daniel (1977), "Bits, Bytes, and IBP”, Bioscience 27: 385.

Brown, James H. and Diane Davidson (1977), "Competition between Seed-eating Rodents and Ants in Desert Ecosystems", Science 196: 880-882.

Dambacher, Jeffrey, Luh, Hang-Kwang, Li, Hiram, and Philippe Rossignol (2003), "Qualitative Stability and Ambiguity in Model Ecosystems", American Naturalist 161: 876-888.

Fishwick, Paul and Paul Luker (eds.) (1991), Qualitative Simulation Modeling and Analysis. New York: Springer Verlag.

Gantmacher, F. (1960), Matrix Theory. Vol. 2. New York: Chelsea Publishing Company.

Jeffries, Clark (1974), "Qualitative Stability and Digraphs in Model Ecosystems", Ecology 55: 1415-1419.

Levins, Richard (1966), "The Strategy of Model Building in Population Biology", American Scientist 54: 421-431.

- (1968), Evolution in Changing Environments. Princeton: Princeton University Press.

(1974), "Qualitative Analysis of Partially Specified Systems", Annals of the New York Academy of Sciences 231: 123-138.

(1975), "Evolution in Communities Near Equilibrium", in Cody, Michael and Jared Diamond (eds.) Ecology and Evolution of Communities. Cambridge: Belknap Press, 16-51.

(1993), "A Response to Orzack and Sober: Formal Analysis and the Fluidity of Science”, Quarterly Review of Biology 68: 547-555.

(1998), "Qualitative Mathematics for Understanding, Prediction, and Intervention in Complex Ecosystems", in Rapport, David; Robert Contanza; Paul Epstein; Connie Gaudet; and Richard Levins (eds.) Ecosystem Health. Malden: Blackwell Science, Inc., 178-204. 
Lewontin, Richard (1969), "The Meaning of Stability", Brookhaven Symposium in Biology 22: 13-24.

Logofet, Dmitrii (1993), Matrices and Graphs: Stability Problems in Mathematical Ecology. Ann Arbor: CRC Press.

Lyapunov, Aleksandr ([1892], 1992), The General Problem of the Stability of Motion. London: Taylor and Francis.

Morgan, Mary and Margaret Morrison (eds.) (1999), Models as Mediators. Cambridge: Cambridge University Press.

Odenbaugh, Jay (2003), "Complex Systems, Tradeoffs, and Theoretical Population Biology: Richard Levin's 'Strategy of Model Building in Population Biology' Revisited", Philosophy of Science 70: 1496-1507.

Orzack, Steven (1990), "The Comparitive Biology of Second Sex Ratio Evolution Within a Natural Population of a Parasitic Wasp, Nasonia Vetripennis", Genetics 124: 385-396.

Orzack, Steven and Elliot Sober (1993), "A Critical Assessment of Levins's The Strategy of Model Building in Population Biology (1966)." Quarterly Review of Biology 68: 533-546.

Puccia, Charles and Richard Levins (1985), Qualitative Modeling of Complex Systems. Cambridge: Harvard University Press.

Quirk, James and Richard Ruppert (1965), "Qualitative Economics and the Stability of Equilibrium”, Review of Economic Studies 32: 311-326.

Sarkar, Sahotra and Justin Garson (2004), "Multiple Criterion Synchronization (MCS) for Conservation Area Network Design: The Use of Non-Dominated Alternative Sets", Conservation and Society (in press).

Simon, Herbert (1991) "Forward", in Fishwick, Paul and Paul Lucker (eds.) Qualitative Simulation Modeling and Analysis. New York: Springer Verlag, v-vii.

Sober, Elliot (1983), “Equilibrium Explanation”, Philosophical Studies 43: 201-210.

Trout, J. D. (2002), "Scientific Explanation and the Sense of Understanding", Philosophy of Science 69: 212-233.

Werren, John (1980), "Sex Ratio Adaptations of Local Mate Competition in a Parasitic Wasp", Science 208: 1157-1159. 
Wimsatt, William (1970), "Some Problems with the Concept of 'Feedback", in PSA 1970, vol. 1. Boston: Philosophy of Science Association, 241-256.

(1987), "False Models as Means to Truer Theories", in Nitecki, Matthew and Antoni Hoffman (eds.) Neutral Models in Biology. New York: Oxford University Press, 23-55.

------ (2001), "Richard Levins as Philosophical Revolutionary", Biology and Philosophy 16: 103-108.

Winsberg, Eric (2001), "Simulations, Models, and Theories: Complex Physical Systems and Their Representations", in PSA 2001, vol. 2 East Lansing: Philosophy of Science Association, 442-454.

Wright, Sewall (1921), "Correlation and Causation", Journal of Agricultural Research 20: $557-585$. 


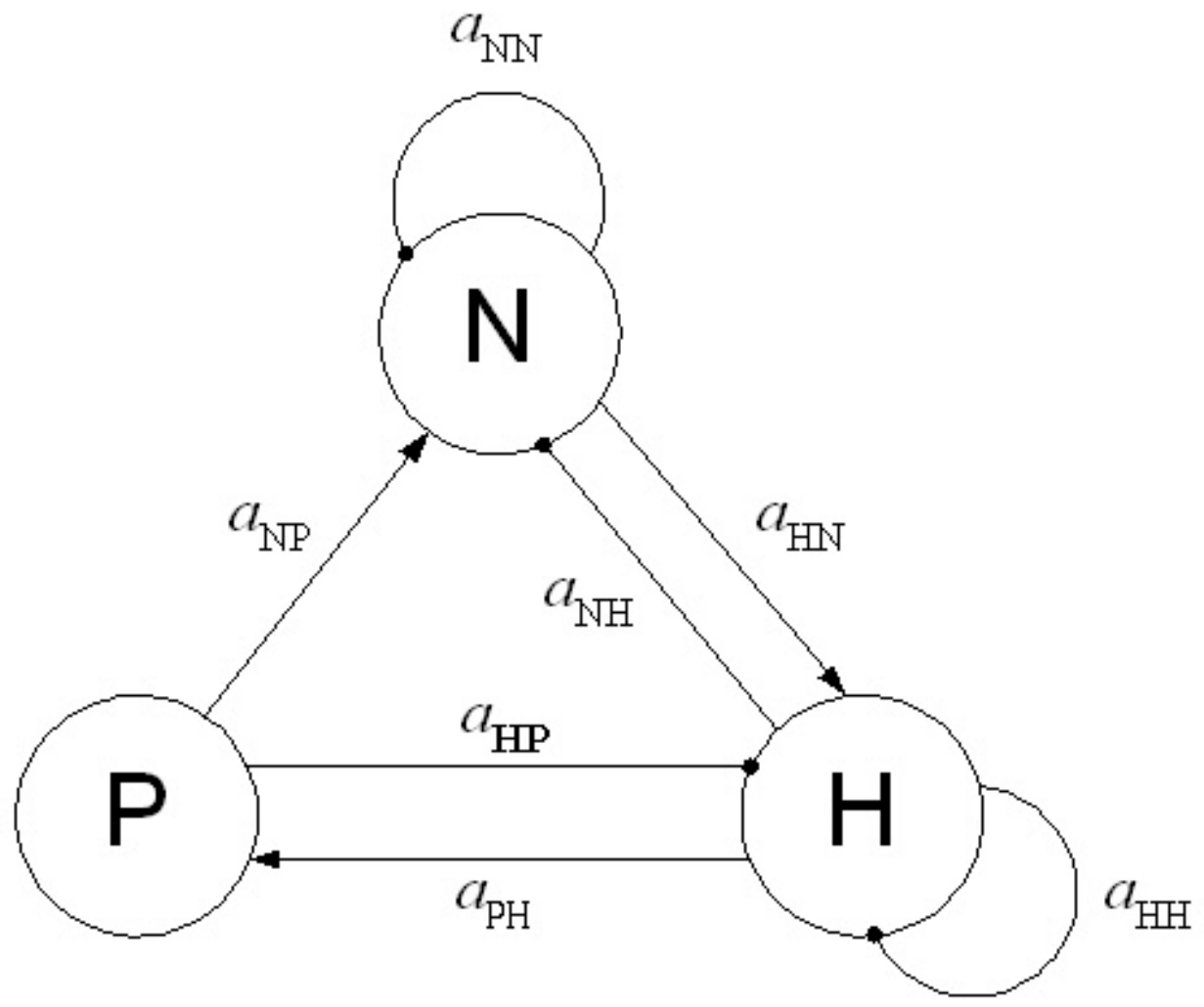

Figure 1. 